\title{
Evaluation des traits de vie d'une espèce endémique du Maroc (Salmo trutta macrostigma, Dumeril, 1858) dans une rivière du Moyen Atlas du Maroc: Oued Sidi Rachid
}

\author{
El Hassan ABBA ${ }^{1 *}$, D. NBELGHYTI ${ }^{1}$, R. EL AYADI ${ }^{2}$ et M. BENABID ${ }^{3}$ \\ ${ }^{1}$ Laboratoire de l'UFR de Parasitologie Comparée et d'Océanographie, Faculté des Sciences, \\ Université Ibn Tofail, B.P. 133, Kénitra 14000 - Maroc. \\ ${ }^{2}$ Laboratoire de biodiversité et des ressources naturelles, Département de Biologie, \\ Faculté des Sciences, Université Ibn Tofail, B.P. 133, Kénitra 14000 - Maroc. \\ ${ }^{3}$ Centre National d'Hydrobiologie et de Pisciculture (CNHP). B.P.11-Azrou 53100-Maroc. \\ *Auteur correspondant,E-mail:abbaelhassan@hotmail.com
}

\section{RESUME}

Cette étude a pour objectif la contribution à la détermination de la qualité physicochimique des eaux de l'Oued Sidi Rachid au Moyen Atlas où vit une espèce endémique du Maroc : la truite de rivière (Salmo trutta macrostigma, Duméril, 1858). L'étude des paramètres essentiels pour la vie de ce salmonidé au cours de la période comprise entre le mois de mai 2007 et le mois d'avril 2008 montre que la température, le potentiel Hydrogène $(\mathrm{pH})$ et les concentrations des nitrites témoignent de la bonne qualité des eaux de l'Oued Sidi Rachid et permettent de classer ce site parmi les sites à vocation salmonicole. Les concentrations des orthophosphates sont très faibles et ne témoignent d'aucune source de pollution organique. Les concentrations des indicateurs de la dureté reflètent la nature géologique (carbonatée) de la région. Les résultats obtenus montrent bien que les eaux de l'Oued Sidi Rachid assurent à la truite de rivière un bon habitat écologique favorable à son développement et à sa reproduction.

(C) 2012 International Formulae Group. All rights reserved.

Mots clés : Qualité physicochimique, Truite de rivière, Oued Sidi Rachid, Maroc.

\section{INTRODUCTION}

L'évolution des populations piscicoles est directement liée aux variations naturelles ou artificielles de l'habitat. Sous le terme de qualité de l'eau, on désigne les principaux paramètres physicochimiques qui agissent de diverses manières sur la biologie des peuplements piscicoles dans un milieu aquatique, ces paramètres peuvent interagir soit d'une façon synergique, soit d'une façon antagoniste.

Dans le cadre de la connaissance des traits de vie des populations de truite 
commune, espèce endémique du Maroc (Salmo trutta macrostigma) (Dumeril, 1858) au Moyen Atlas au Maroc, et afin de déterminer les impacts des bassins de salmoniculture de Ras El Ma situés en amont du cours d'eau, une étude des paramètres physicochimiques des eaux a été menée entre le mois de mai 2007 et le mois d'avril 2008. La détermination des caractéristiques de l'habitat lotique permettra d'évaluer sa qualité avant toute intervention dans le cadre des aménagements que les responsables peuvent faire pour l'amélioration des conditions de vie des poissons et par conséquent l'augmentation de la production des géniteurs destinés à la reproduction artificielle de la truite commune.

\section{MATERIEL ET METHODES}

\section{Présentation du site d'étude}

L'Oued Sidi Rachid de coordonnées géographiques $5^{\circ} 9^{\prime} \mathrm{N}$ et $33^{\circ} 28^{\prime} \mathrm{W}$ se trouve à une altitude de $1620 \mathrm{~m}$ au sud-est de la ville d'Ifrane (Fig.1). C'est l'un des rares ruisseaux permanents de la région d'Ifrane. L'Oued coule sur une distance de quelques kilomètres (moins de $3 \mathrm{Km}$ ) avant de se diviser en plusieurs petits canaux (Seguias). En plus de son rôle piscicole, les eaux de l'Oued sont utilisées dans le domaine d'irrigation des terres agricoles d'Ougmes de la commune d'Ait Ali Ouyakoub, province d'Ifrane. Du point de vue morphométrie, l'Oued présente une faible largeur qui peut varier de $2 \mathrm{~m}$ à 6 $\mathrm{m}$, et une profondeur qui ne dépasse pas $1 \mathrm{~m}$ en général. L'Oued Sidi Rachid, se situe dans la dépression de Ras El Ma qui correspond à un repli synclinal. Cette dépression fait partie du haut plateau de Tazoualt-Tamrabta qui est l'un des quatre éléments structuraux du causse d'Ifrane. Du point de vue faciès lithologique, le causse d'Ifrane est caractérisé par des roches carbonatées du Lias (Martin, 1981).
Présentation des stations d'échantillonnage

$\mathrm{Vu}$ la petite longueur d'écoulement de

l'Oued Sidi Rachid, deux stations d'échantillonnages seulement ont été choisies pour la détermination de la qualité physicochimique des eaux du cours d'eau (Figure 1). La station SA1 se trouve près de la zone de captage des eaux destinées à l'eau potable de la ville d'Azrou, l'autre station SA2 se trouve dans la partie avale où se déversent les eaux en provenance des bassins de salmoniculture de la station de Ras El Ma. Le choix des stations a été fait dans le but de déterminer non pas seulement la qualité physicochimique de l'eau où vit la truite commune, espèce endémique du Maroc, mais également l'impact que peut présenter les bassins de salmoniculture et les activités anthropiques liées généralement à l'agriculture au niveau de la deuxième station (Aguilar, 2004).

\section{Méthodologie d'échantillonnage}

L'échantillonnage de l'eau a été fait au cours de la période comprise entre mai 2007 et avril 2008. Les prélèvements sont effectués d'une façon instantanée entre $10 \mathrm{~h}$ et $12 \mathrm{~h}$ du matin avec une fréquence mensuelle.

Quelques paramètres sont mesurés in situ (température, oxygène dissous, potentiel hydrogène $(\mathrm{pH})$ et conductivité) par des appareils de mesure portatifs de type Orion. Des échantillons d'eau sont ramenés au laboratoire dans une glacière pour d'autres analyses (alcalinité, duretés, chlorures, sulfates orthophosphates et nitrites) effectuées par des méthodes homologuées (Rodier, 1996) dans le laboratoire de la qualité des eaux appartenant au Centre National d'Hydrobiologie et de Pisciculture d'Azrou (CNHP). 


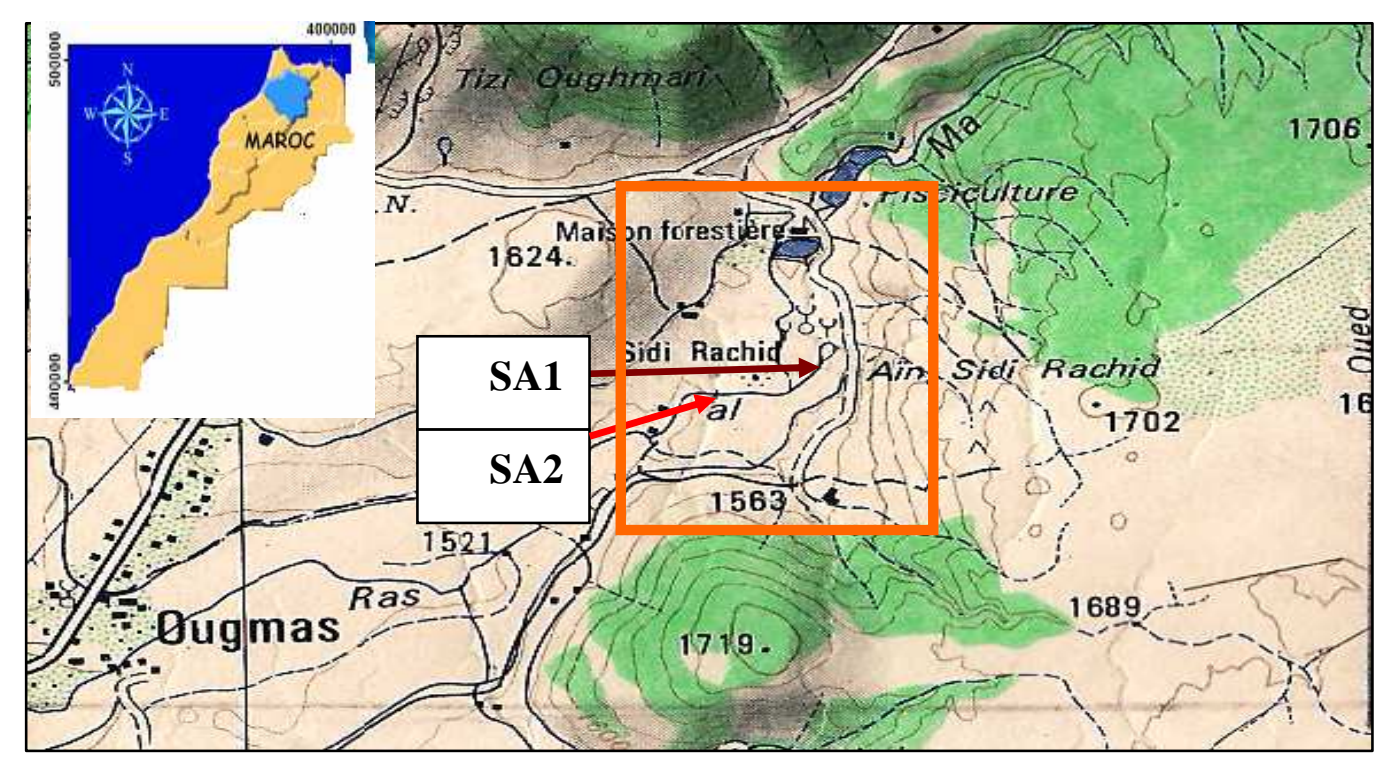

Figure 1 : Situation du site et des stations d'étude (Extrait de la carte d'Azrou. E : 1/50.OOO ; Division de la carte, 1974, Maroc).

\section{RESULTATS ET DISCUSSION}

Les résultats du suivi des descripteurs de la qualité physicochimique de l'écosystème aquatique, l'Oued Sidi Rachid où vit la truite commune, espèce endémique du Maroc, au cours de la période d'étude comprise entre mai 2007 et avril 2008 sont consignés dans le Tableau 1.

La température du milieu de vie constitue un facteur écologique primaire qui agit sur la biologie et le comportement des truites (Gouraud et al., 1997). Les températures maximales obtenues entre le mois de mai 2007 et le mois d'avril 2008 sont respectivement $13,8^{\circ} \mathrm{C}$ pour la station SA1 et $15,7{ }^{\circ} \mathrm{C}$ pour SA2. Par contre, la température minimale est la même pour les deux stations $\left(11,7{ }^{\circ} \mathrm{C}\right)$. Pour la température moyenne annuelle, elle est de $12,22{ }^{\circ} \mathrm{C}$ pour SA1 et $13,93{ }^{\circ} \mathrm{C}$ pour SA2. Pour la truite fario, la température enregistrée au cours de toute la période d'étude constitue un paramètre de vie favorable pour son développement puisque la température maximale n'atteint pas $20{ }^{\circ} \mathrm{C}$ (Bagliniere, 1999) et que la plage des températures optimales pour la croissance se situe autour de $16{ }^{\circ} \mathrm{C}$ (Launey et Vandeputte, 2004).

Les concentrations en oxygène au niveau de la station SA2 sont élevées que celles enregistrées au niveau de SA1; cette différence s'explique par le phénomène de photosynthèse assuré par la présence d'une végétation bien développée (Abba et al., 2008) ainsi qu'une vitesse d'écoulement de l'eau plus importante au niveau de SA2. Les taux d'oxygénation enregistrés au niveau des deux stations sont suffisants et ne présentent aucun déficit pour la truite commune dont les exigences sont importantes (Bagliniere, 1999 ; Norme de l'Union Européen (NUE), 2006) puisque les concentrations minimales dépassent $6 \mathrm{mg} / \mathrm{l}$ au niveau des deux stations.

La moyenne annuelle du potentiel Hydrogène $(\mathrm{pH})$ est de 7,38 au niveau de SA1 et 7,61 au niveau de SA2, le pH maximal ne dépasse pas 8,5 dans les deux stations. Vis-àvis de la vie des poissons comme la truite commune, les eaux de l'Oued Sidi Rachid sont de bonne qualité puisque les valeurs du critère de bonne qualité des eaux pour la vie aquatique sont comprises entre 6,5 et 9 
(Painchaud, 1997; Norme de l'Union Européen (NUE), 2006).

Les valeurs de la conductivité électrique des eaux au niveau des deux stations évoluent de la même façon et ne présentent pas d'écart notable. La moyenne observée est de l'ordre de $295 \mu \mathrm{S} / \mathrm{cm}$ au niveau de SA1 et $292 \mu \mathrm{S} / \mathrm{cm}$ au niveau de SA2. Pour les eaux salmonicoles, la conductivité doit être comprise entre 150 et $750 \mu \mathrm{S} / \mathrm{cm}$ (Alabaster et Liyod, 1980).

Les valeurs maximales de la dureté calcique sont $52,12 \mathrm{mg} / \mathrm{L}$ au niveau de SA1 et $75,35 \mathrm{mg} / \mathrm{L}$ au niveau de SA2 et sont observées au cours du mois de janvier pour SA1 et décembre pour SA2. Ces valeurs coïncident avec les périodes où l'activité photosynthétique n'est pas importante ce qui augmente la solubilité des carbonates de calcium. Pour les concentrations moyennes, elles sont de l'ordre de $35,68 \mathrm{mg} / \mathrm{L}$ pour $\mathrm{SA} 1$ et $43,35 \mathrm{mg} / \mathrm{L}$ pour $\mathrm{SA} 2$. La valeur minimale enregistrée au niveau de SA1 est supérieure à celle enregistrée au niveau de SA2.

La dureté calcique au niveau de SA2 est légèrement supérieure à celle enregistrée au niveau de SA1.

Les valeurs moyennes en ion magnésium sont respectivement de 26,23 $\mathrm{mg} / \mathrm{L}$ et $21,23 \mathrm{mg} / \mathrm{L}$ au niveau de SA1 et SA2. Pour les poissons d'eau douce dont les besoins en ion magnésium sont très faibles, les eaux de l'Oued Sidi Rachid peuvent leur assurer en partie leur besoin puisque la concentration des ions magnésium de ses eaux est supérieure à 3 $\mathrm{mg} / \mathrm{L}$.

La dureté totale (ou titre hydrotimétrique TH) d'une eau, est principalement, l'expression de la teneur en calcium et magnésium dans l'eau. Les valeurs minimales et les valeurs moyennes annuelles ne montrent pas de différence notable entre les deux stations. D'une manière générale, les eaux de l'Oued Sidi Rachid présentent une dureté totale influencée principalement par les ions calcium. Ceci s'explique par la nature géologique du bassin versant dont la lithologie est de nature carbonatée.
Les carbonates et les hydrogénocarbonates évoluent en fonction du $\mathrm{pH}$ de l'eau qui est lui même en fonction de l'activité photosynthétique et de la respiration. Pour les eaux de l'Oued Sidi Rachid, les valeurs maximales et moyennes ne présentent pas de différences notables entre SA1 et SA2 et elles sont de l'ordre de $35 \mathrm{mg} / \mathrm{L}$ et $25 \mathrm{mg} / \mathrm{L}$ respectivement. Pour les carbonates, les concentrations sont nulles puisque le $\mathrm{pH}$ des eaux de l'Oued Sidi Rachid est inférieur à 8,3.

Les concentrations moyennes des chlorures sont $41,71 \mathrm{mg} / \mathrm{L}$ pour SA1 et 36,23 $\mathrm{mg} / \mathrm{L}$ pour SA2. L'augmentation des teneurs en chlorures au niveau de SA1 peut avoir comme origine le phénomène de chloration qui se fait au niveau de la station de captage des eaux potables.

Les concentrations des orthophosphates varient d'un mois à l'autre au niveau des deux stations. Les valeurs maximales, respectivement de $1,42 \mathrm{mg} / \mathrm{L}$ en $\mathrm{SA} 1$ et de $3,16 \mathrm{mg} / \mathrm{L}$ en $\mathrm{SA} 2$, ont été enregistrées au cours des mois de septembre et août 2007. Les valeurs moyennes annuelles sont de 0,57 $\mathrm{mg} / \mathrm{L}$ pour $\mathrm{SA} 1$ et $0,8 \mathrm{mg} / \mathrm{L}$ pour $\mathrm{SA} 2$. Les valeurs importantes au niveau de SA2 peuvent s'expliquer par le fait que cette station est la plus proche des cultures qui entourent l'Oued (lessivage des engrais) et des habitations.

Pour les sulfates, les concentrations sont très faibles et sont comprises entre 0 $\mathrm{mg} / \mathrm{L}$ et $1 \mathrm{mg} / \mathrm{l}$, ces concentrations témoignent de l'absence des roches salifères qui peuvent être à l'origine des concentrations plus importantes.

Les valeurs maximales et les valeurs moyennes des nitrites sont les mêmes au niveau des deux stations. Elles sont respectivement de l'ordre de $0,014 \mathrm{mg} / \mathrm{L}$ au niveau de SA1 et $0,01 \mathrm{mg} / \mathrm{L}$ au niveau de $\mathrm{SA} 2$. Pour les concentrations minimales, on constate une légère hausse au niveau de SA2. D'une façon générale, les concentrations des nitrites ne dépassent pas les concentrations guides des eaux salmonicoles qui sont de l'ordre de $0,01 \mathrm{mg} / \mathrm{L}$. 
Tableau 1 : Résultats des paramètres physicochimiques des eaux au niveau des deux stations d'études SA1 et SA2.

\begin{tabular}{llllllllc}
\hline \multicolumn{1}{c}{ VARIABLES } & \multicolumn{2}{c}{ MOYENNES } & \multicolumn{2}{c}{ MAX } & \multicolumn{2}{c}{ MIN } & \multicolumn{2}{c}{ ECART TYPE } \\
\hline & SA1 & SA2 & SA1 & SA2 & SA1 & SA2 & SA1 & SA2 \\
$\mathrm{T}^{\circ}\left({ }^{\circ} \mathrm{C}\right)$ & 12,08 & 13,79 & 13,8 & 15,7 & 11,7 & 11,8 & 0,55 & 1,4 \\
$\mathrm{pH}$ & 7,3 & 7,8 & 8,5 & 8,06 & 6,3 & 7,11 & 0,52 & 0,25 \\
Conductivité $\mu \mathrm{S} / \mathrm{Cm}$ & 273,44 & 271,65 & 556 & 547 & 117,9 & 114,1 & 196,68 & 197,33 \\
Oxygène $(\mathrm{mg} / \mathrm{L})$ & 8,34 & 10,04 & 10,46 & 13,32 & 6,4 & 7,38 & 1,12 & 1,91 \\
$\mathrm{NO} 2(\mathrm{mg} / \mathrm{L})$ & 0,0108 & 0,011 & 0,014 & 0,014 & 0,004 & 0,009 & 0,0028 & 0,0016 \\
Orthophosphate(mg/L) & 0,5 & 0,61 & 1,42 & 3,16 & 0,08 & 0,08 & 0,46 & 0,93 \\
$\mathrm{TAC}(\mathrm{mg} / \mathrm{L})$ & 24,5 & 25,04 & 34,2 & 35,39 & 13,42 & 18,92 & 6,26 & 5,16 \\
$\mathrm{Mg}(\mathrm{mg} / \mathrm{L})$ & 25,75 & 22,05 & 39,39 & 29,23 & 10,7 & 10,21 & 7,76 & 6,61 \\
$\mathrm{Ca}(\mathrm{mg} / \mathrm{L})$ & 34,31 & 41,27 & 52,12 & 75,35 & 24,02 & 20,84 & 8,66 & 14,45 \\
$\mathrm{TH}(\mathrm{mg} / \mathrm{L})$ & 78,44 & 72,75 & 96 & 104 & 48,8 & 48 & 14,53 & 15,55 \\
$\mathrm{Cl}(\mathrm{mg} / \mathrm{L})$ & 40,75 & 34,81 & 53,25 & 53,25 & 35,5 & 17,75 & 7,08 & 12,15 \\
\hline
\end{tabular}

\section{Conclusion}

La qualité de l'eau et par conséquent sa productivité et sa vocation piscicole dépendent d'un certain nombre de descripteurs conventionnels de la qualité physique et chimique des eaux du milieu d'étude. Le suivi des paramètres phares pour la vie des salmonidés comme la température, le taux d'oxygène dissous, le potentiel Hydrogène $(\mathrm{pH})$ ainsi que les nitrites des eaux de l'Oued Sidi Rachid témoignent d'une bonne qualité des eaux et permettent de classer toujours les eaux parmi les eaux de première classe à vocation salmonicole. Ces caractéristiques offrent à la truite commune un milieu écologique favorable pour sa croissance et sa reproduction. Les indicateurs de la pollution organique sont très faibles et ne montrent pas de différence notable entre les deux stations. Les concentrations des éléments de la dureté reflètent la nature géologique de la région qui est de type carbonaté.

\section{REFERENCES}

Abba H, Benabid M, El Ayadi R, Nassali H, El ibaoui H. 2008, Contribution à l'étude physicochimique de l'écosystème lacustre Dayet Aoua du Maroc. Afrique Science, 4(2): 11-31.

Aguilar Ibarra A. 2004. Les peuplements de poissons comme outil pour la gestion de la qualité environnementale du réseau hydrographique de la Garonne. Thèse $\mathrm{N}^{\circ}$ 2087, INP ENSAT, p.133.

Alabaster JS, Liyod R. 1980. Water Quality Criteria for Freshwater Fish. Butterworths: London; 297p.

Gouraud V. 1994. Modélisation de la dynamique d'une population de truite commune. Introduction d'une mobilité des poissons due aux variations de la qualité d'habitat du milieu. ENGREF, Mémoire de fin d'études, p. 215.

Launney S, Vandeputte M. 2004. Quelle gestion génétique de la domestication chez les poissons ? INRA. Prod. Anim., 17: 237-242. 
Martin J. 1981. Le Moyen Atlas Central. Editions du Service Géologique du Maroc : Rabat, Maroc.

Norme de l'Union Européen. 2006. Journal Officiel de l'Union Européenne. Directive 006/44/CE du Parlement Européen et du Conseil 2006, concernant la qualité des eaux douces ayant besoin d'être protégées ou améliorées pour être aptes à la vie des poissons.
Painchaud J. 1997. La qualité de l'eau des rivières du Québec : état et tendances. Ministère de l'Environnement et de la Faune, Direction des écosystèmes aquatiques, Québec, 58 p.

Rodier J. 1996. L'Analyse de l'Eau Naturelle, Eaux Résiduaires, Eau de Mer (8 $8^{\mathrm{eme} e}$ édn). Dunod : Paris; 1-1383. 